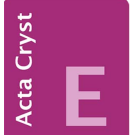

\section{Crystal structure of $\mathrm{CdSO}_{4}\left(\mathrm{H}_{2} \mathrm{O}\right)$ : a redetermination}

\author{
Chatphorn Theppitak ${ }^{\mathrm{a}}$ and Kittipong Chainok ${ }^{\mathrm{b} *}$ \\ ${ }^{a}$ Department of Chemistry, Faculty of Science, Naresuan University, Muang, \\ Phitsanulok, 65000, Thailand, and ${ }^{\mathbf{b}}$ Department of Physics, Faculty of Science and \\ Technology, Thammasat University, Khlong Luang, Pathum Thani, 12120, Thailand. \\ *Correspondence e-mail: kc@tu.ac.th
}

Received 31 August 2015; accepted 9 September 2015

Edited by M. Weil, Vienna University of Technology, Austria

The crystal structure of the title compound, cadmium sulfate monohydrate or poly[( $\mu_{2}$-aqua $)\left(\mu_{4}\right.$-sulfato $)$ cadmium $]$, was redetermined based on modern CMOS (complementary metal oxide silicon) data. In comparison with the previous study [Bregeault \& Herpin (1970). Bull. Soc. Fr. Mineral. Cristallogr. 93, 37-42], all non-H atoms were refined with anisotropic displacement parameters and the hydrogen-bonding pattern unambiguously established due to location of the hydrogen atoms. In addition, a significant improvement in terms of precision and accuracy was achieved. In the crystal, the $\mathrm{Cd}^{2+}$ cation is coordinated by four $\mathrm{O}$ atoms of four sulfate anions and two $\mathrm{O}$ atoms of water molecules, forming a distorted octahedral trans- $\left[\mathrm{CdO}_{6}\right]$ polyhedron. Each sulfate anion bridges four $\mathrm{Cd}^{2+}$ cations and each water molecule bridges two $\mathrm{Cd}^{2+}$ cations, leading to the formation of a threedimensional framework, with $\mathrm{Cd} \cdots \mathrm{Cd}$ separations in the range 4.0757 (2)-6.4462 (3) $\AA$. O-H ‥ O hydrogen-bonding interactions of medium strength between the coordinating water molecules and sulfate anions consolidate the crystal packing.

Keywords: crystal structure; redetermination; cadmium sulfate monohydrate; hydrothermal synthesis; hydrogen bonding.

CCDC reference: 1423357

\section{Related literature}

For the previous report on the structure of the title compound, see: Bregeault \& Herpin (1970).

\section{Experimental}

2.1. Crystal data

$\mathrm{CdSO}_{4}\left(\mathrm{H}_{2} \mathrm{O}\right)$
Monoclinic, $P 2_{1} / c$

$a=7.6195(3) \mathrm{A}$

$b=7.4517(3) \AA$

$c=8.1457(3) \AA$

$\beta=122.244(1)^{\circ}$

$V=391.17(3) \AA^{3}$

\subsection{Data collection}

Bruker APEXII D8 QUEST CMOS diffractometer

Absorption correction: multi-scan (SADABS; Bruker, 2014)

$T_{\min }=0.701, T_{\max }=0.746$

\subsection{Refinement}

$R\left[F^{2}>2 \sigma\left(F^{2}\right)\right]=0.012$

$w R\left(F^{2}\right)=0.026$

$S=1.18$

1004 reflections

72 parameters

Table 1

Hydrogen-bond geometry $\left(\AA{ }^{\circ}\right)$.

\begin{tabular}{lllll}
\hline$D-\mathrm{H} \cdots A$ & $D-\mathrm{H}$ & $\mathrm{H} \cdots A$ & $D \cdots A$ & $D-\mathrm{H} \cdots A$ \\
\hline $\mathrm{O} 5-\mathrm{H} 5 A \cdots \mathrm{O} 3^{\mathrm{i}}$ & $0.82(2)$ & $1.88(2)$ & $2.6958(17)$ & $170(3)$ \\
$\mathrm{O} 5-\mathrm{H} 5 B \cdots \mathrm{O} 2^{\mathrm{ii}}$ & $0.86(2)$ & $1.90(2)$ & $2.7530(17)$ & $173(2)$ \\
\hline
\end{tabular}

Symmetry codes: (i) $-x+1, y-\frac{1}{2},-z+\frac{1}{2}$; (ii) $x, y-1, z$.

Table 2

Comparison of bond lengths $(\AA)$ in the current and the previous (Bregeault \& Herpin, 1970) refinement of cadmium sulfate monohydrate.

For the previous refinement: $a=7.607, b=7.541, c=8.186 \AA, \beta=121.86^{\circ}$ and reliability index $R=0.12$.

\begin{tabular}{lll}
\hline Bond & Current refinement & Previous refinement \\
\hline $\mathrm{Cd} 1-\mathrm{O} 1^{\mathrm{i}}$ & $2.2417(12)$ & $2.21(5)$ \\
$\mathrm{Cd} 1-\mathrm{O} 2^{\text {ii }}$ & $2.2530(13)$ & $2.27(3)$ \\
$\mathrm{Cd} 1-\mathrm{O} 3$ & $2.2421(12)$ & $2.36(5)$ \\
$\mathrm{Cd} 1-\mathrm{O} 4^{\mathrm{iii}}$ & $2.3112(12)$ & $2.33(3)$ \\
$\mathrm{Cd} 1-\mathrm{O} 5^{\mathrm{i}}$ & $2.3210(12)$ & $2.24(3)$ \\
$\mathrm{Cd} 1-\mathrm{O} 5$ & $2.4024(12)$ & $2.33(3)$ \\
$\mathrm{S} 1-\mathrm{O} 1$ & $1.4703(12)$ & $1.50(4)$ \\
$\mathrm{S} 1-\mathrm{O} 2$ & $1.4845(12)$ & $1.62(6)$ \\
$\mathrm{S} 1-\mathrm{O} 3$ & $1.4831(12)$ & $1.45(3)$ \\
$\mathrm{S} 1-\mathrm{O} 4$ & $1.4584(12)$ & $1.42(4)$ \\
\hline Symmetry codes: (i) $x,-y+\frac{1}{2}, z+\frac{1}{2},(\mathrm{ii})-x,-y+1,-z ;(\mathrm{iii})-x+1, y-\frac{1}{2},-z+\frac{1}{2}$
\end{tabular}

Symmetry codes: (i) $x,-y+\frac{1}{2}, z+\frac{1}{2}$; (ii) $-x,-y+1,-z$; (iii) $-x+1, y-\frac{1}{2},-z+\frac{1}{2}$.

Data collection: APEX2 (Bruker, 2014); cell refinement: SAINT (Bruker, 2014); data reduction: SAINT; method used to solve structure: coordinates taken from previous refinement; program(s) used to refine structure: SHELXL2014 (Sheldrick, 2015); molecular graphics: OLEX2 (Dolomanov et al., 2009); software used to prepare material for publication: OLEX2 and publCIF (Westrip, 2010).

\section{Acknowledgements}

The authors gratefully acknowledge the financial support provided by Thammasat University Research Fund under the TU Research Scholar. 
Supporting information for this paper is available from the IUCr electronic archives (Reference: WM5211).

\section{References}

Bregeault, J. M. \& Herpin, P. (1970). Bull. Soc. Fr. Mineral. Cristallogr. 93, $37-$ 42.
Bruker (2014). APEX2, SADABS and SAINT. Bruker AXS Inc., Madison, Wisconsin, USA.

Dolomanov, O. V., Bourhis, L. J., Gildea, R. J., Howard, J. A. K. \& Puschmann, H. (2009). J. Appl. Cryst. 42, 339-341.

Sheldrick, G. M. (2015). Acta Cryst. C71, 3-8.

Westrip, S. P. (2010). J. Appl. Cryst. 43, 920-925. 


\section{supporting information}

Acta Cryst. (2015). E71, i8-i9 [doi:10.1107/S2056989015016904]

\section{Crystal structure of $\mathrm{CdSO}_{4}\left(\mathrm{H}_{2} \mathrm{O}\right)$ : a redetermination}

\section{Chatphorn Theppitak and Kittipong Chainok}

\section{S1. Synthesis and crystallization}

The title compound was obtained serendipitously. $\mathrm{CdSO}_{4} \cdot 8 / 3 \mathrm{H}_{2} \mathrm{O}(0.256 \mathrm{~g}, 1 \mathrm{mmol})$ and 3,6-di-2-pyridyl-1,2,4,5-tetrazine $(0.236 \mathrm{~g}, 1 \mathrm{mmol})$ dissolved in water $(5 \mathrm{ml})$ were added to a $23 \mathrm{ml}$ Teflon-lined autoclave and heated at $356 \mathrm{~K}$ for 5 days. The product was collected by filtration, washed with water and air-dried. Colourless block-shaped crystals of the title compound suitable for X-ray analysis were isolated.

\section{S2. Refinement}

The same cell setting and atom numbering scheme as in the previous refinement (Bregeault \& Herpin, 1970) were used. Starting coordinates for the atoms were also taken from the previous model. Hydrogen atoms of the water molecules were located from difference Fourier maps and were refined with an $\mathrm{O}-\mathrm{H}$ distance restraint of 0.85 (2) $\AA$.

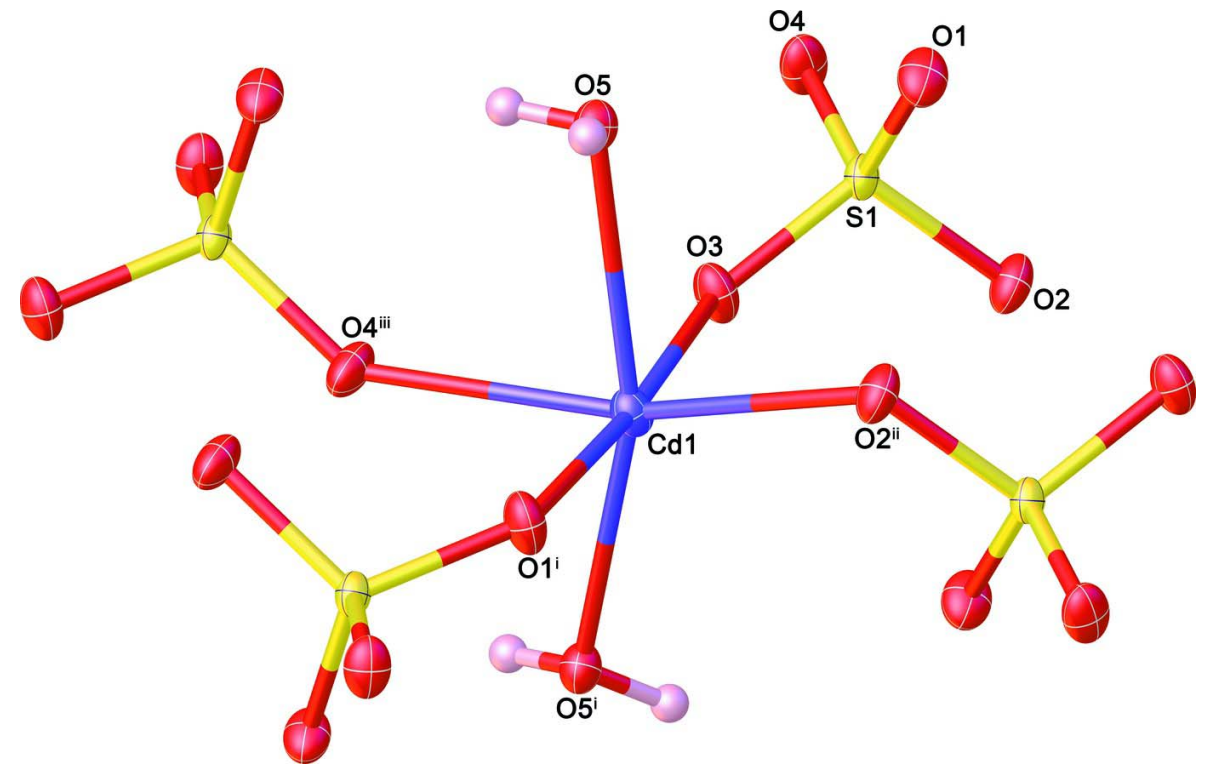

\section{Figure 1}

The coordination sphere around the $\mathrm{Cd}^{2+}$ cation with displacement ellipsoids drawn at the $50 \%$ probability level. [Symmetry codes: (i) $x, 1 / 2-y, 1 / 2+z$; (ii) $-x, 1-y,-z$; (iii) $1-x,-1 / 2+y, 1 / 2-z$ ] 


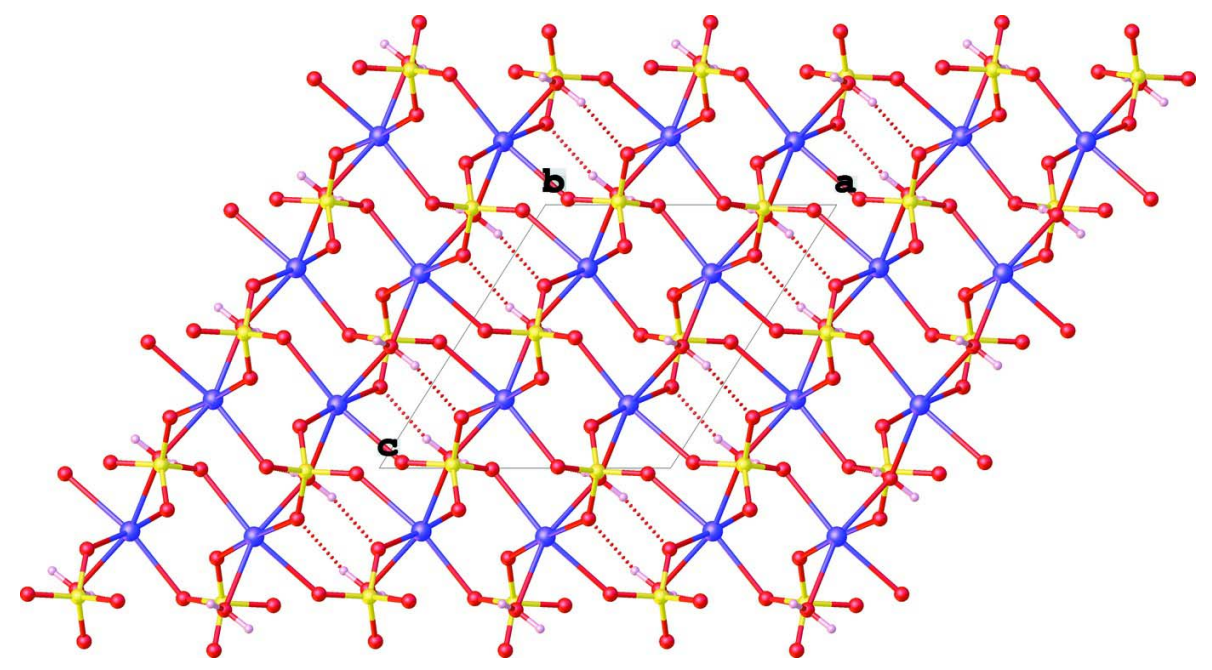

\section{Figure 2}

The three-dimensional framework structure of the title compound in a view along the $b$ axis. Dashed lines indicate intermolecular $\mathrm{O}-\mathrm{H} \cdots \mathrm{O}$ hydrogen-bonding interactions.

\section{Poly $\left[\left(\mu_{2}\right.\right.$-aqua $)\left(\mu_{4}\right.$-sulfato $)$ cadmium $]$}

\section{Crystal data}

$\mathrm{CdSO}_{4}\left(\mathrm{H}_{2} \mathrm{O}\right)$

$M_{r}=226.48$

Monoclinic, $P 2{ }_{1} / c$

$a=7.6195(3) \AA$

$b=7.4517(3) \AA$

$c=8.1457(3) \AA$

$\beta=122.244(1)^{\circ}$

$V=391.17(3) \AA^{3}$

$Z=4$

\section{Data collection}

Bruker APEXII D8 QUEST CMOS

diffractometer

Radiation source: microfocus sealed x-ray tube, Incoatec I $\mu$ us

GraphiteDouble Bounce Multilayer Mirror monochromator

Detector resolution: 10.5 pixels $\mathrm{mm}^{-1}$

$\omega$ and $\varphi$ scans

Absorption correction: multi-scan

(SADABS; Bruker, 2014)

\section{Refinement}

Refinement on $F^{2}$

Least-squares matrix: full

$R\left[F^{2}>2 \sigma\left(F^{2}\right)\right]=0.012$

$w R\left(F^{2}\right)=0.026$

$S=1.18$

1004 reflections

72 parameters
$F(000)=424$

$D_{\mathrm{x}}=3.846 \mathrm{Mg} \mathrm{m}^{-3}$

Mo $K \alpha$ radiation, $\lambda=0.71073 \AA$

Cell parameters from 9894 reflections

$\theta=3.2-30.5^{\circ}$

$\mu=6.01 \mathrm{~mm}^{-1}$

$T=296 \mathrm{~K}$

Block, colourless

$0.26 \times 0.22 \times 0.22 \mathrm{~mm}$

$T_{\min }=0.701, T_{\max }=0.746$

17459 measured reflections

1004 independent reflections

958 reflections with $I>2 \sigma(I)$

$R_{\text {int }}=0.023$

$\theta_{\text {max }}=28.7^{\circ}, \theta_{\min }=3.2^{\circ}$

$h=-10 \rightarrow 10$

$k=-10 \rightarrow 10$

$l=-10 \rightarrow 10$

2 restraints

Primary atom site location: structure-invariant direct methods

Hydrogen site location: difference Fourier map

All $\mathrm{H}$-atom parameters refined

$w=1 /\left[\sigma^{2}\left(F_{\mathrm{o}}^{2}\right)+(0.0107 P)^{2}+0.2955 P\right]$

where $P=\left(F_{\mathrm{o}}^{2}+2 F_{\mathrm{c}}^{2}\right) / 3$ 
$(\Delta / \sigma)_{\max }=0.001$

$\Delta \rho_{\max }=0.28 \mathrm{e} \AA^{-3}$

Special details

Experimental. SADABS was used for absorption correction. wR2(int) was 0.0449 before and 0.0357 after correction. The Ratio of minimum to maximum transmission is 0.9396 . The $\lambda / 2$ correction factor is 0.00150 .

Geometry. All e.s.d.'s (except the e.s.d. in the dihedral angle between two 1.s. planes) are estimated using the full covariance matrix. The cell e.s.d.'s are taken into account individually in the estimation of e.s.d.'s in distances, angles and torsion angles; correlations between e.s.d.'s in cell parameters are only used when they are defined by crystal symmetry. An approximate (isotropic) treatment of cell e.s.d.'s is used for estimating e.s.d.'s involving 1.s. planes.

Fractional atomic coordinates and isotropic or equivalent isotropic displacement parameters $\left(\AA^{2}\right)$

\begin{tabular}{lllll}
\hline & $x$ & $y$ & $z$ & $U_{\text {iso }} * / U_{\text {eq }}$ \\
\hline Cd1 & $0.21887(2)$ & $0.26013(2)$ & $0.26053(2)$ & $0.01330(5)$ \\
S1 & $0.25524(6)$ & $0.61729(5)$ & $0.01217(5)$ & $0.01018(8)$ \\
O1 & $0.13754(18)$ & $0.50022(16)$ & $-0.15811(17)$ & $0.0183(2)$ \\
O2 & $0.1103(2)$ & $0.75977(15)$ & $-0.00480(19)$ & $0.0169(3)$ \\
O3 & $0.32756(18)$ & $0.51255(16)$ & $0.19211(17)$ & $0.0168(2)$ \\
O4 & $0.43393(18)$ & $0.69973(18)$ & $0.02148(18)$ & $0.0184(2)$ \\
O5 & $0.27603(19)$ & $0.09752(16)$ & $0.03844(17)$ & $0.0140(2)$ \\
H5A & $0.400(3)$ & $0.074(4)$ & $0.110(3)$ & $0.038(7)^{*}$ \\
H5B & $0.216(3)$ & $-0.005(2)$ & $0.016(3)$ & $0.023(6)^{*}$ \\
\hline
\end{tabular}

Atomic displacement parameters $\left(\AA^{2}\right)$

\begin{tabular}{lllllll}
\hline & $U^{11}$ & $U^{22}$ & $U^{33}$ & $U^{12}$ & $U^{13}$ & $U^{23}$ \\
\hline Cd1 & $0.01176(7)$ & $0.01373(7)$ & $0.01200(7)$ & $-0.00165(4)$ & $0.00472(5)$ & $0.00087(4)$ \\
S1 & $0.00809(16)$ & $0.00898(17)$ & $0.01063(17)$ & $0.00001(13)$ & $0.00310(14)$ & $0.00041(13)$ \\
O1 & $0.0156(6)$ & $0.0170(6)$ & $0.0158(6)$ & $-0.0004(5)$ & $0.0041(5)$ & $-0.0054(5)$ \\
O2 & $0.0122(6)$ & $0.0134(6)$ & $0.0206(6)$ & $0.0027(4)$ & $0.0058(5)$ & $-0.0018(4)$ \\
O3 & $0.0155(6)$ & $0.0153(6)$ & $0.0147(5)$ & $-0.0019(5)$ & $0.0048(5)$ & $0.0046(4)$ \\
O4 & $0.0101(5)$ & $0.0224(6)$ & $0.0198(6)$ & $-0.0022(5)$ & $0.0061(5)$ & $0.0047(5)$ \\
O5 & $0.0120(5)$ & $0.0133(5)$ & $0.0145(5)$ & $-0.0008(4)$ & $0.0055(5)$ & $-0.0001(4)$ \\
\hline
\end{tabular}

Geometric parameters $\left(\AA,{ }^{\circ}\right)$

\begin{tabular}{llll}
\hline $\mathrm{Cd} 1-\mathrm{O} 1^{\mathrm{i}}$ & $2.2417(12)$ & $\mathrm{S} 1-\mathrm{O} 3$ & $1.4831(12)$ \\
$\mathrm{Cd} 1-\mathrm{O} 2^{\mathrm{ii}}$ & $2.2530(13)$ & $\mathrm{S} 1-\mathrm{O} 4$ & $1.4584(12)$ \\
$\mathrm{Cd} 1-\mathrm{O} 3$ & $2.2421(12)$ & $\mathrm{O} 1-\mathrm{Cd} 1^{\mathrm{iv}}$ & $2.2417(12)$ \\
$\mathrm{Cd} 1-\mathrm{O} 4^{\mathrm{iii}}$ & $2.3112(12)$ & $\mathrm{O} 2-\mathrm{Cd} 1^{1 i}$ & $2.2530(13)$ \\
$\mathrm{Cd} 1-\mathrm{O} 5^{\mathrm{i}}$ & $2.3210(12)$ & $\mathrm{O} 4-\mathrm{Cd} 1^{\mathrm{v}}$ & $2.3112(12)$ \\
$\mathrm{Cd} 1-\mathrm{O} 5$ & $2.4024(12)$ & $\mathrm{O} 5-\mathrm{Cd} 1^{\mathrm{iv}}$ & $2.3211(12)$ \\
$\mathrm{S} 1-\mathrm{O} 1$ & $1.4703(12)$ & $\mathrm{O} 5-\mathrm{H} 5 \mathrm{~A}$ & $0.822(17)$ \\
$\mathrm{S} 1-\mathrm{O} 2$ & $1.4845(12)$ & $\mathrm{O} 5-\mathrm{H} 5 \mathrm{~B}$ & $0.859(16)$ \\
& & & $109.76(7)$ \\
$\mathrm{O} 1-\mathrm{Cd} 1-\mathrm{O} 2^{\mathrm{ii}}$ & $82.50(4)$ & $\mathrm{O} 1-\mathrm{S} 1-\mathrm{O} 3$ & $109.52(8)$ \\
$\mathrm{O} 1^{\mathrm{i}}-\mathrm{Cd} 1-\mathrm{O} 3$ & $175.24(4)$ & $\mathrm{O} 3-\mathrm{S} 1-\mathrm{O} 2$ & $112.43(8)$ \\
$\mathrm{O} 1-\mathrm{Cd} 1-\mathrm{O} 4^{\mathrm{iii}}$ & $89.31(5)$ & $\mathrm{O} 4-\mathrm{S} 1-\mathrm{O} 1$ &
\end{tabular}




\begin{tabular}{|c|c|c|c|}
\hline $\mathrm{O} 1^{\mathrm{i}}-\mathrm{Cd} 1-\mathrm{O} 5^{\mathrm{i}}$ & $92.57(4)$ & $\mathrm{O} 4-\mathrm{S} 1-\mathrm{O} 2$ & $109.35(7)$ \\
\hline $\mathrm{O} 1^{\mathrm{i}}-\mathrm{Cd} 1-\mathrm{O} 5$ & $88.62(4)$ & $\mathrm{O} 4-\mathrm{S} 1-\mathrm{O} 3$ & $109.03(7)$ \\
\hline $\mathrm{O} 2^{\mathrm{ii}}-\mathrm{Cd} 1-\mathrm{O} 4^{\mathrm{iii}}$ & $161.94(4)$ & $\mathrm{S} 1-\mathrm{O} 1-\mathrm{Cd} 1^{\mathrm{iv}}$ & $131.81(7)$ \\
\hline $\mathrm{O} 2^{\mathrm{ii}}-\mathrm{Cd} 1-\mathrm{O} 5$ & $80.22(4)$ & $\mathrm{S} 1-\mathrm{O} 2-\mathrm{Cd} 1^{\mathrm{ii}}$ & $116.42(7)$ \\
\hline $\mathrm{O} 2^{\mathrm{ii}}-\mathrm{Cd} 1-\mathrm{O} 5^{\mathrm{i}}$ & $117.97(4)$ & $\mathrm{S} 1-\mathrm{O} 3-\mathrm{Cd} 1$ & $134.06(7)$ \\
\hline $\mathrm{O} 3-\mathrm{Cd} 1-\mathrm{O} 2^{\mathrm{ii}}$ & $101.65(4)$ & $\mathrm{S} 1-\mathrm{O} 4-\mathrm{Cd} 1^{\mathrm{v}}$ & $140.91(8)$ \\
\hline $\mathrm{O} 3-\mathrm{Cd} 1-\mathrm{O} 4^{\mathrm{iii}}$ & $86.05(4)$ & $\mathrm{Cd} 1^{\mathrm{iv}}-\mathrm{O} 5-\mathrm{Cd} 1$ & $119.27(5)$ \\
\hline $\mathrm{O} 3-\mathrm{Cd} 1-\mathrm{O} 5$ & $89.80(4)$ & $\mathrm{Cd} 1^{\mathrm{iv}}-\mathrm{O} 5-\mathrm{H} 5 \mathrm{~A}$ & $109.7(18)$ \\
\hline $\mathrm{O} 3-\mathrm{Cd} 1-\mathrm{O}^{\mathrm{i}}$ & $87.54(4)$ & $\mathrm{Cd} 1-\mathrm{O} 5-\mathrm{H} 5 \mathrm{~A}$ & $99.9(18)$ \\
\hline $\mathrm{O} 4^{\mathrm{iii}}-\mathrm{Cd} 1-\mathrm{O} 5^{\mathrm{i}}$ & $78.31(4)$ & $\mathrm{Cd}^{\mathrm{iv}}-\mathrm{O} 5-\mathrm{H} 5 \mathrm{~B}$ & $113.3(14)$ \\
\hline $\mathrm{O} 4^{\mathrm{iii}}-\mathrm{Cd} 1-\mathrm{O} 5$ & $83.53(4)$ & $\mathrm{Cd} 1-\mathrm{O} 5-\mathrm{H} 5 \mathrm{~B}$ & $108.6(14)$ \\
\hline $\mathrm{O} 55^{\mathrm{i}}-\mathrm{Cd} 1-\mathrm{O} 5$ & $161.78(6)$ & $\mathrm{H} 5 \mathrm{~A}-\mathrm{O} 5-\mathrm{H} 5 \mathrm{~B}$ & $104(2)$ \\
\hline $\mathrm{O} 1-\mathrm{S} 1-\mathrm{O} 2$ & $106.70(7)$ & & \\
\hline $\mathrm{O} 1-\mathrm{S} 1-\mathrm{O} 2-\mathrm{Cd} 1^{\mathrm{ii}}$ & $2.46(10)$ & $\mathrm{O} 3-\mathrm{S} 1-\mathrm{O} 1-\mathrm{Cd}^{\mathrm{iv}}$ & $-54.81(11)$ \\
\hline $\mathrm{O} 1-\mathrm{S} 1-\mathrm{O} 3-\mathrm{Cd} 1$ & $-23.82(12)$ & $\mathrm{O} 3-\mathrm{S} 1-\mathrm{O} 2-\mathrm{Cd} 1^{\mathrm{ii}}$ & $-116.29(8)$ \\
\hline $\mathrm{O} 1-\mathrm{S} 1-\mathrm{O} 4-\mathrm{Cd}^{\mathrm{v}}$ & $-135.57(12)$ & $\mathrm{O} 3-\mathrm{S} 1-\mathrm{O} 4-\mathrm{Cd}^{\mathrm{v}}$ & $-13.61(15)$ \\
\hline $\mathrm{O} 2-\mathrm{S} 1-\mathrm{O} 1-\mathrm{Cd} 1^{\mathrm{iv}}$ & $-173.39(9)$ & $\mathrm{O} 4-\mathrm{S} 1-\mathrm{O} 1-\mathrm{Cd} 1^{\mathrm{iv}}$ & $66.74(12)$ \\
\hline $\mathrm{O} 2-\mathrm{S} 1-\mathrm{O} 3-\mathrm{Cd} 1$ & $93.01(11)$ & $\mathrm{O} 4-\mathrm{S} 1-\mathrm{O} 2-\mathrm{Cd} 1^{\mathrm{ii}}$ & $124.30(8)$ \\
\hline $\mathrm{O} 2-\mathrm{S} 1-\mathrm{O} 4-\mathrm{Cd}^{\mathrm{v}}$ & $106.10(13)$ & $\mathrm{O} 4-\mathrm{S} 1-\mathrm{O} 3-\mathrm{Cd} 1$ & $-147.38(10)$ \\
\hline
\end{tabular}

Symmetry codes: (i) $x,-y+1 / 2, z+1 / 2$; (ii) $-x,-y+1,-z$; (iii) $-x+1, y-1 / 2,-z+1 / 2$; (iv) $x,-y+1 / 2, z-1 / 2$; (v) $-x+1, y+1 / 2,-z+1 / 2$.

Hydrogen-bond geometry $\left(\AA,{ }^{\circ}\right)$

\begin{tabular}{lllll}
\hline$D-\mathrm{H} \cdots A$ & $D-\mathrm{H}$ & $\mathrm{H} \cdots A$ & $D \cdots A$ & $D-\mathrm{H}^{\cdots} A$ \\
\hline $\mathrm{O} 5-\mathrm{H} 5 A \cdots \mathrm{O} 3^{\mathrm{iii}}$ & $0.82(2)$ & $1.88(2)$ & $2.6958(17)$ & $170(3)$ \\
$\mathrm{O} 5-\mathrm{H} 5 B \cdots \mathrm{O} 2^{\text {vi }}$ & $0.86(2)$ & $1.90(2)$ & $2.7530(17)$ & $173(2)$ \\
\hline
\end{tabular}

Symmetry codes: (iii) $-x+1, y-1 / 2,-z+1 / 2$; (vi) $x, y-1, z$. 